\title{
Modified culture protocol for testing anti-fibrotic drugs using precision-cut liver slices
}

Theerut Luangmonkong1, Peter Olinga ${ }^{2 *}$

${ }^{1}$ Department of Pharmacology, Faculty of Pharmacy, Mahidol University, Thailand 2Department of Pharmaceutical

Technology and Biopharmacy, Faculty of Science and Engineering, University of Groningen, the Netherlands

\section{*Corresponding author:}

Peter Olinga

p.olinga@rug.nl

\section{KEYWORDS:}

Anti-fibrotic; Culture method; Culture protocol; Precision-cut liver slices; LY2109761.

https://www.pharmacy.mahidol.ac.th/journal/ (C) Faculty of Pharmacy, Mahidol University (Thailand) 2020

\begin{abstract}
Precision-cut liver slices (PCLS) have been widely utilized in various multicellular studies due to liver architecture and intercellular communication remain preserved. In studying antifibrotic drugs using PCLS, the culture method is currently based on a protocol for applications in drug metabolism and toxicity. This published protocol requires the pre-incubation step to incubate PCLS in a drug-free condition. However, this lag period may limit the effect of anti-fibrotic drugs. Therefore, we aimed to study whether a modification of culture protocol provided benefits in studying anti-fibrotic drugs. In this study, PCLS were cultured with LY2109761, the representative anti-fibrotic drug, by either published or modified protocol. Tissue viability and gene/protein expression of fibrosis-related markers of cultured PCLS were assessed. We found that, despite slightly depressed viability (ATP/protein of PCLS was $71-77 \%$ of those cultured by the published protocol), spontaneous fibrosis which is a distinctive feature of PCLS was greatly pronounced by the modified culture protocol. As a result, this novel culture protocol demonstrated apparent inhibitory effect of LY2109761 on the gene expression of fibrosis markers, particularly heat shock protein 47 and alpha smooth muscle actin (the inhibition was higher by $44 \%$ and $23 \%$ when compared to those cultured by published protocol respectively). This modified culture protocol could be a useful alternative for testing anti-fibrotic drugs using PCLS. Besides the differences in tissue viability and spontaneous fibrosis, the modified culture protocol might reduce time and cost of experiment.
\end{abstract}

\section{INTRODUCTION}

Precision-cut liver slices (PCLS) have been widely utilized in a myriad of preclinical study due to liver architecture and intercellular communication remain preserved. These unique features of PCLS permit an application of this ex vivo model for a study of multicellular diseases including liver fibrosis ${ }^{1-3}$. Over the past decade, multiple anti-fibrotic drugs with various mechanisms of action were tested using PCLS, and some of them demonstrated an attractive anti-fibrotic efficacy warranting further in-depth researches ${ }^{4-6}$. Nevertheless, until now, the protocol to culture PCLS in the testing of anti-fibrotic drugs is solely based on a conventional protocol for an application in drug metabolism and toxicity ${ }^{7}$. According to this published protocol, a pre-incubation step which is 
the incubation of PCLS in drug-free culture medium for $1 \mathrm{~h}$ was recommended. This preincubation step aims to eliminate undesirable enzymes and waste products during the processing of liver tissue in temperature-controlled condition ${ }^{7}$. Thus, the viability of PCLS was effectively recovered. Unfortunately, a previous study reported that a marker of fibrosis, that is heat shock protein 47, was promptly up-regulated during the preincubation step, indicating preceding expression of this fibrosis marker prior to PCLS-drug incubation 5. This finding may imply that the anti-fibrotic effects of tested drugs could be pronounced when PCLS are instantaneously cultured without this drug-free period.

In this study, we aimed to evaluate whether a modification of culture protocol by immediately providing drug-PCLS exposure impact the effect of anti-fibrotic drugs. LY2109761, a small molecule inhibitor of transforming growth factor beta (TGF- $\beta$ ) which previously exhibited a marked inhibitory effect on the expression of fibrosis markers in PCLS, mainly via blocking Smad2 phosphorylation which is the key process of TGF- $\beta$ signaling, was used as the representative anti-fibrotic drug ${ }^{8}$.

\section{MATERIALS AND METHODS}

\subsection{Animal}

Male Wistar rats (Charles River, Sulzfeld, Germany), 12-16 weeks old were used for the study. The rats were housed under a $12 \mathrm{~h}$ light/dark cycle in a temperature/humidity- controlled room with food and water ad libitum. The study was performed after acclimatization for at least 7 days. For the euthanization, the rats were anaesthetized using isoflurane before decapitation. The liver was isolated and harvested for the preparation of PCLS. The study protocol which was approved by the Animal Ethical Committee of the University of Groningen complied with the ARRIVE guidelines ${ }^{9}$, and carried out in accordance with the Directive 2010/63/EU on the protection of animals used for scientific purposes.

\subsection{Preparation of PCLS}

Freshly harvested livers were stored in ice-cold University of Wisconsin (UW) organ preservation solution (DuPont Critical Care, Waukegan, US). PCLS were prepared in a KrebsHenseleit buffer supplemented with $25 \mathrm{mM}$ Dglucose (Merck, Darmstadt, Germany), $25 \mathrm{mM}$ $\mathrm{NaHCO}_{3}$ (Merck), 10 mM HEPES (MP Biomedicals, Aurora, USA), and saturated with carbogen $\left(95 \% \mathrm{O}_{2} / 5 \% \mathrm{CO}_{2}\right)$, using a Krumdieck tissue slicer as previously described ${ }^{7}$. Prepared PCLS, with a diameter of $5 \mathrm{~mm}$ and a thickness of $250 \mathrm{~mm}$, were kept in ice-cold UW organ preservation solution again prior to starting the culture.

\subsection{Experimental design and culture protocol}

Williams Medium E supplemented with Glutamax $^{\mathrm{TM}}$ (Invitrogen, Paisly, UK), $25 \mathrm{mM}$ glucose and $50 \mathrm{mg} / \mathrm{mL}$ gentamycin (Invitrogen)

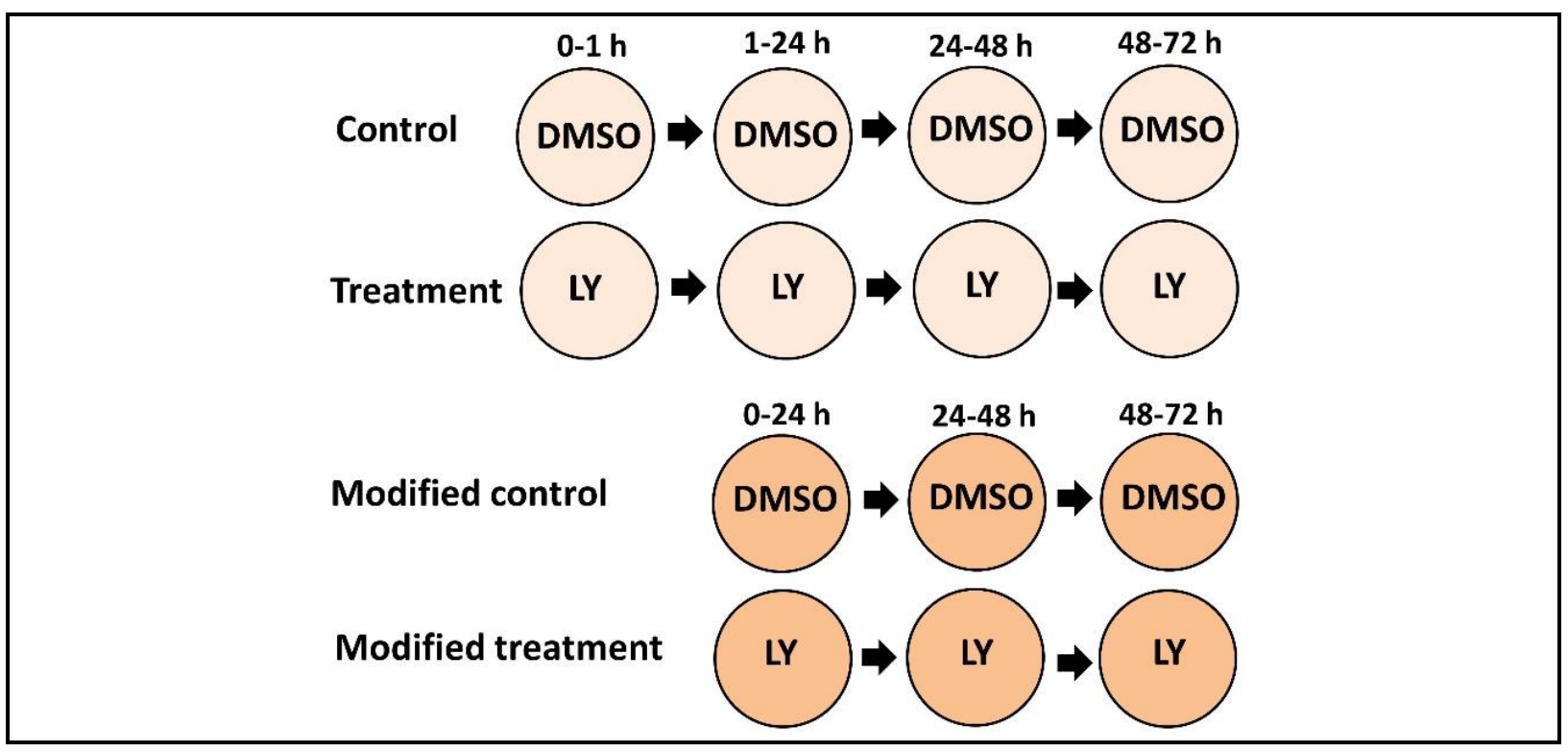

Figure 1. The culture protocol and treatment of each group. DMSO=dimethyl sulfoxide, LY=LY2109761. 
in a volume of $1.3 \mathrm{~mL}$ was used as culture medium. PCLS were randomly divided into 4 groups (Figure 1). The pre-incubation step was performed by incubating PCLS in the culture medium without LY2109761 or dimethyl sulfoxide (DMSO) for $1 \mathrm{~h}$ before transferring to the treatment conditions. Stock solution of $5 \mathrm{mM}$ LY2109761 (Selleckchem, Munich, Germany) prepared in DMSO was added to the culture medium to yield $20 \mu \mathrm{M}$ in the treatment groups, while control groups contained DMSO only. The concentration of DMSO was less than $0.4 \%$ volume/volume throughout experiments. PCLS, both during pre-incubation and incubation with DMSO or LY2109761, were cultured at 37 ${ }^{\circ} \mathrm{C}$ under continuous supply of $80 \% \mathrm{O}_{2} / 5 \% \mathrm{CO}_{2}$ in 12 -wells plates with gently shaking. The culture medium of each treatment was refreshed every 24h. After $72 \mathrm{~h}$, treated PCLS were snap frozen and stored at $-80{ }^{\circ} \mathrm{C}$ until analysis.

\subsection{ATP and protein determination}

Viability of PCLS was determined by measuring ATP levels ${ }^{7}$. Briefly, PCLS were transferred to a solution containing $70 \%$ ethanol and $2 \mathrm{mM}$ EDTA, snap frozen, and stored at -80 ${ }^{\circ} \mathrm{C}$ until analysis. After thawing, ATP of the PCLS was determined using the ATP bioluminescence kit (Roche Diagnostics). Protein of PCLS was determined using the BioRad DC Protein Assay (Bio-Rad, California, US). ATP values were corrected for total soluble protein content of each sample estimated.

\subsection{Total RNA isolation and real-time quantitative polymerase chain reaction}

Total RNA was isolated form PCLS using the RNeasy Mini Kit (Qiagen, Venlo, the Netherlands). Gene expression of fibrosis markers was assessed by real-time quantitative PCR ${ }^{4,8}$. Reverse transcription of RNA was performed using the Reverse Transcription System (Promega, Leiden, the Netherlands). The mRNA levels of collagen type I-alpha 1 (Collal), heat shock protein 47(Hsp47), alpha smooth muscle actin $(\alpha \mathrm{Sma})$, and plasminogen activator inhibitor 1 (Pai-1) were detected using specific primers (Sigma; Table 1) with the SensiMIX SYBR kit (Bioline, Taunton, US). The PCR reaction was performed using a 7900HT Real Time PCR apparatus (Applied Biosystems) with 1 cycle of 10 minutes $/ 95^{\circ} \mathrm{C}$ followed by 45 cycles of 15 seconds $/ 95^{\circ} \mathrm{C}$ and 25 seconds $/ 60^{\circ} \mathrm{C}$ following by a dissociation stage. Glyceraldehyde 3-phosphate dehydrogenase (Gapdh) was used as reference gene. Relative mRNA expression levels were calculated as fold change using the $2^{-\Delta \Delta \mathrm{CT}}$ method.

\subsection{Western blotting}

Phosphorylated Smad2 (pSmad2) and total Smad2 (tSmad2) were assessed by Western blot $^{4,8}$. Following homogenization of PCLS in the lysis buffer containing a mixture of phosphatase inhibitors $(30 \mathrm{mM}$ Tris- $\mathrm{HCl} \mathrm{pH} 7.4,150 \mathrm{mM}$ $\mathrm{NaCl}, 1 \mu \mathrm{M}$ EDTA, $5.4 \mathrm{mg} / \mathrm{mL}$ Triton X-100, 1\% SDS, $15 \mathrm{mM}$ sodium orthovanadate, $15 \mathrm{mM}$ sodium fluoride, 1 tablet of PhosSTOP ${ }^{\mathrm{TM}}$ (Roche Diagnostics) in $50 \mathrm{~mL}$ of lysis buffer), total soluble protein was separated on $10 \%$ sodium dodecylsulfate polyacrylamide gels. The separated protein was transferred using Trans-Blot Turbo Mini PVDF Transfer Packs (Bio-Rad). Subsequently, the membrane was blocked and incubated with specific antibodies (Antiglyceraldehyde 3-phosphate dehydrogenase, Sigma; Anti-phospho-Smad2 (Ser465/467), Cell Signaling, Massachusetts, US; Anti-Smad2, Abcam, Cambridge, UK). Targeted proteins were visualized with Clarity Western ECL Substrate (Bio-Rad). Gapdh was used as internal control protein.

Table 1. Primer sequences used in quantitative real-time PCR.

\begin{tabular}{|c|c|c|}
\hline Gene & Forward primer & Reverse primer \\
\hline $\begin{array}{l}\boldsymbol{a S m a} \\
\text { (alpha smooth muscle actin) }\end{array}$ & AGCTCTGGTGTGTGACAATGG & GGAGCATCATCACCAGCAAAG \\
\hline $\begin{array}{l}\text { Colla1 } \\
\text { (collagen type I-alpha 1) }\end{array}$ & CCCACCGGCCCTACTG & GACCAGCTTCACCCTTAGCA \\
\hline $\begin{array}{l}\text { Gapdh } \\
\text { (glyceraldehyde 3-phosphate } \\
\text { dehydrogenase) }\end{array}$ & GAACATCATCCCTGCATCCA & CCAGTGAGCTTCCCGTTCA \\
\hline $\begin{array}{l}\text { Hsp47 } \\
\text { (heat shock protein } 47 \text { ) }\end{array}$ & AGACGAGTTGTAGAGTCCAAGAGT & ACCCATGTGTCTCAGGAACCT \\
\hline $\begin{array}{l}\text { Pai-1 } \\
\text { (plasminogen activator inhibitor 1) }\end{array}$ & AACCCAGGCCGACTTCA & CATGCGGGCTGAGACTAGAAT \\
\hline
\end{tabular}



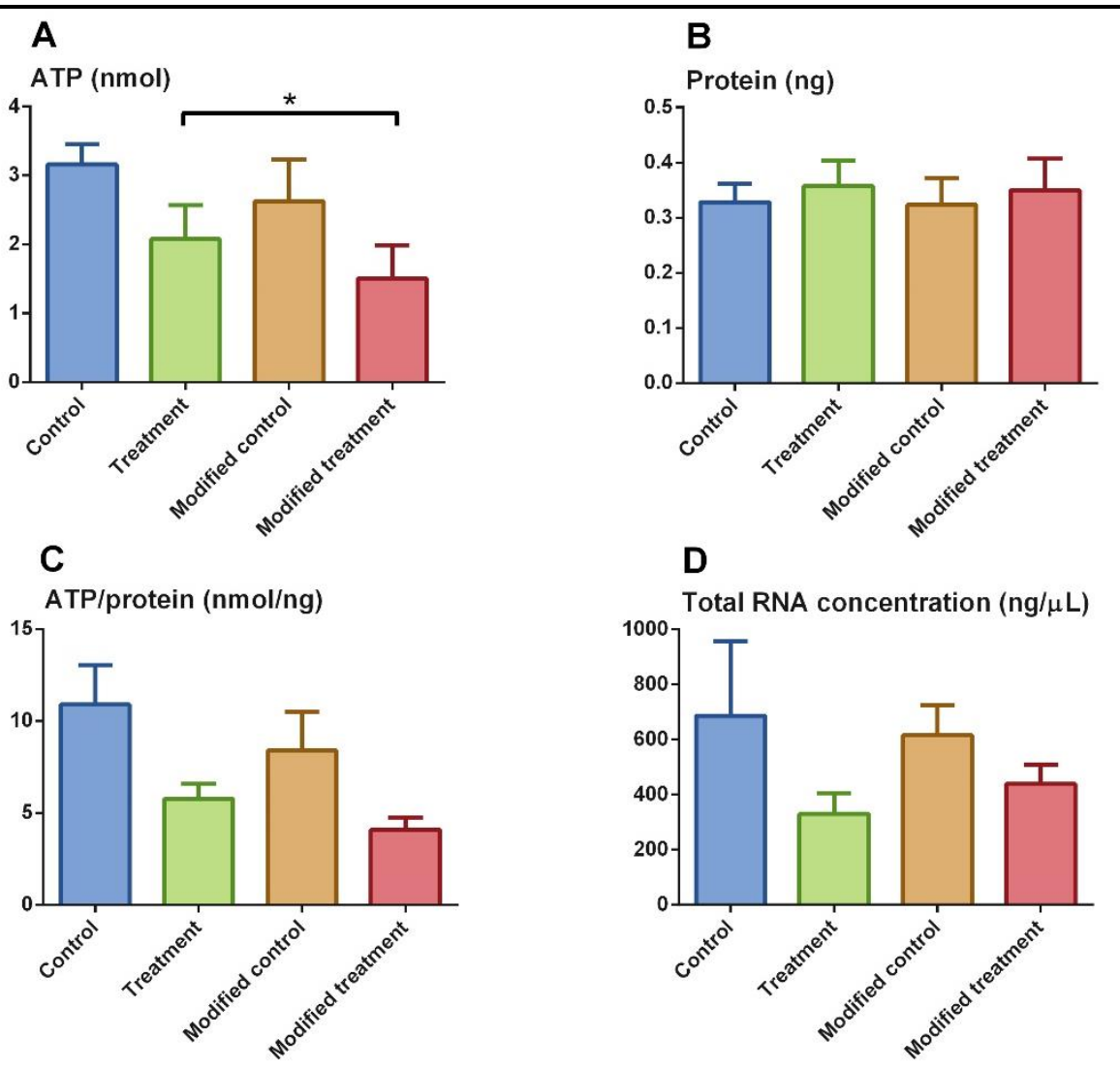

Figure 2. Viability of PCLS in conventional and modified culture protocol for 72h. (A) ATP in nmol per slice, (B) protein in ng per slice, (C) ATP/protein in nmol of ATP per ng of protein per slice, and (D) total RNA concentration in $n g / \mu \mathrm{L}$. Data is expressed as mean $+\mathrm{SEM}(\mathrm{n}=3) . * p<0.05$ when compared to treatment.

\subsection{Statistical analysis}

Each experiment was performed three times $(n=3)$, in which triplicate slices from the same liver were used for individual downstream assays. Results are expressed as means + standard error of the mean (SEM). Analysis of statistical significance was performed using one-way ANOVA for ATP, protein, ATP/protein, and total RNA concentration, and paired $t$-test for gene and protein expression. For the gene expression, $\Delta \mathrm{Ct}$ was used for the calculation of statistical significance. A $p$-value $<0.05$ was considered significant.

\section{RESULTS}

\subsection{Tissue viability}

First, we assessed ATP value and protein content of PCLS to determine whether this modified protocol affects tissue viability. After culturing for $72 \mathrm{~h}$, the ATP value of control was $3.1 \mathrm{nmol}$ per slice while that of modified control was $2.6 \mathrm{nmol}$ per slice $(84 \%$ compared to control). Similarly, the ATP value of treatment and modified treatment were 2.1 and $1.5 \mathrm{nmol}$ per slice ( $71 \%$ compared to treatment), respectively (Figure 2A). The decreased protein contents among modified protocol was not observed, as protein content of both modified control and modified treatment were $97 \%$ compared to control and treatment, respectively (Figure 2B). After normalizing ATP value of PCLS using corresponding protein content, ATP/protein of control was $10.9 \mathrm{nmol} / \mathrm{ng}$ while that of modified control was $8.4 \mathrm{nmol} / \mathrm{ng}$ ( $77 \%$ compared to control). Similarly, the ATP/protein of treatment and modified treatment were 5.8 and $4.1 \mathrm{nmol} / \mathrm{ng}$ (71\% compared to treatment), respectively (Figure 2C). Therefore, it seemed that the modified protocol slightly depressed viability of PCLS. Nevertheless, it should be noted that the value of ATP of PCLS cultured in modified conditions was in line with levels found in previous studies 4,8 .

Considering the effect of anti-fibrotic drug, $20 \mu \mathrm{M}$ LY2109761 tended to diminish the viability of PCLS cultured by both unmodified and modified protocol in a similar extent. The 


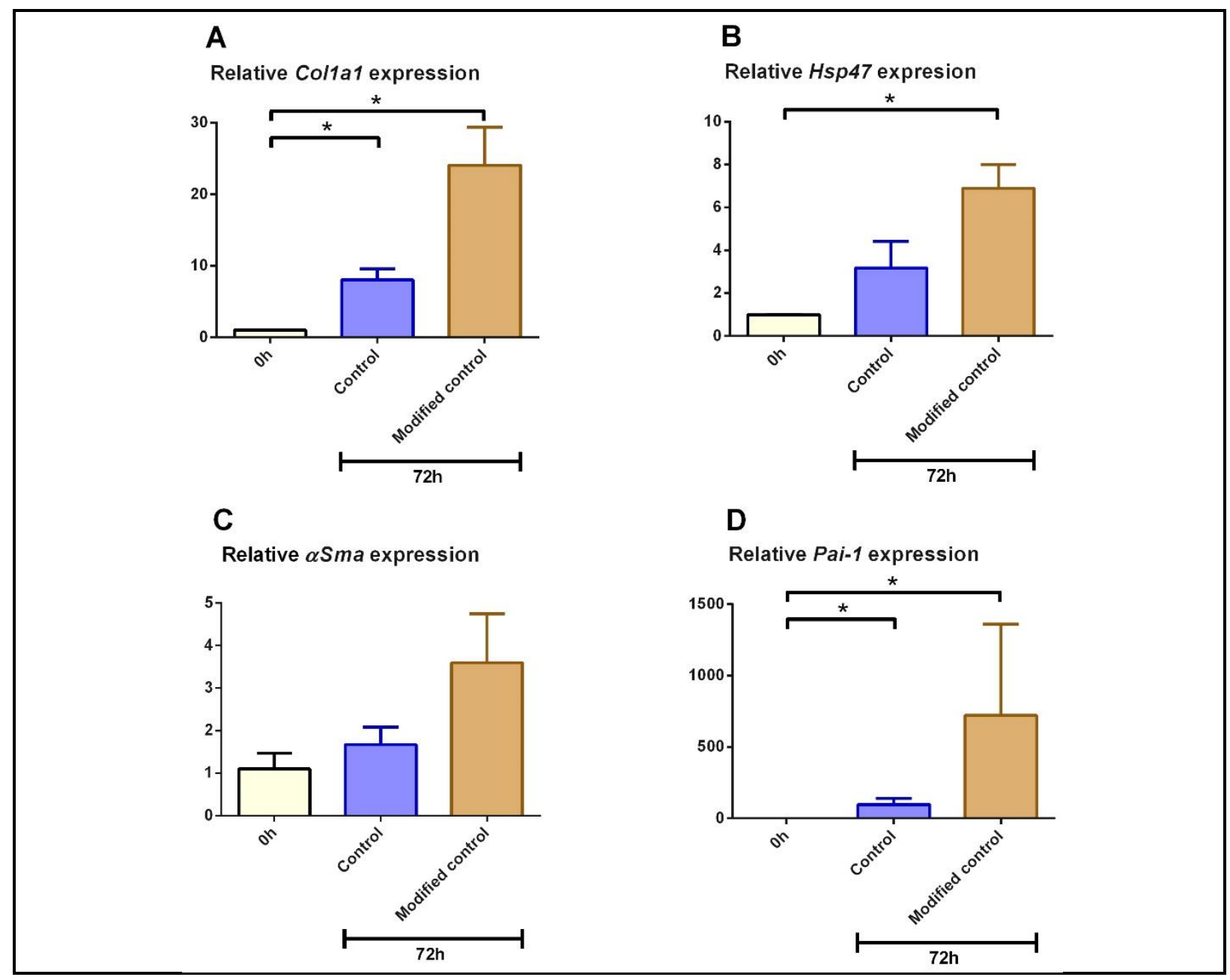

Figure 3. Relative mRNA expression of fibrosis markers of PCLS in control and modified control after culture for 72h. (A) Collagen type I-alpha 1 (Collal), (B) heat shock protein 47 (Hsp47), (C) alpha smooth muscle actin ( $\alpha$ Sma), and (D) plasminogen-activator inhibitor 1 (Pai-1). Data is expressed as mean $+\operatorname{SEM}(\mathrm{n}=3)$. ${ }^{*} p<0.05$ when compared to $0 \mathrm{~h}$.

ATP/protein of treatment and modified treatment were $53 \%$ and $49 \%$ of control and modified control, respectively (Figure 2C). Also, treatment with LY2109761 slightly lowered the concentrations of total RNA isolated from PCLS (Figure 2D). The RNA concentration in control was $686 \mathrm{ng} / \mu \mathrm{L}$ compared to $330 \mathrm{ng} / \mu \mathrm{L}$ in treatment (48\% compared to control). Similarty, the total RNA of modified control was $615 \mathrm{ng} / \mu \mathrm{L}$ compared to $483 \mathrm{ng} / \mu \mathrm{L}$ in modified treatment (79\% compared to modified control). Despite the decrease, the concentrations of RNA were sufficient for subsequent mRNA quantification.

\subsection{Expression of fibrosis markers}

Spontaneous fibrosis, a distinctive feature of PCLS which can be observed by an increased expression of fibrosis markers, was significantly pronounced in the modified protocol after culture for $72 \mathrm{~h}$ (Figure 3). In control, the level of Collal, Hsp47, $\alpha \mathrm{Sma}$, and Pai-1 were 8.0-fold, 3.2-fold, 1.7-fold, and 98.0fold, respectively, when compared to $0 \mathrm{~h}$. This increment was lower than those of modified control which the expression of Collal, Hsp47, oSma, and Pai-1 were 24.1-fold, 6.9-fold, 3.6fold, and 721.3-fold, respectively, when compared to $0 \mathrm{~h}$.

In line with spontaneous fibrosis, the inhibitory effect of LY2109761 on the expression of fibrosis markers was explicit in modified protocol (Figure 4). In treatment, the expression of Collal, Hsp47, $\alpha \mathrm{Sma}$, and Pai-1 were inhibited by $92 \%, 36 \%, 66 \%$, and $74 \%$ respectively, when compared to control. In modified treatment, the mRNA level of Collal, Hsp 47, $\alpha$ Sma, and Pai- 1 were decreased by $98 \%$, $80 \%, 89 \%$, and $84 \%$ respectively, when compared to modified control. 


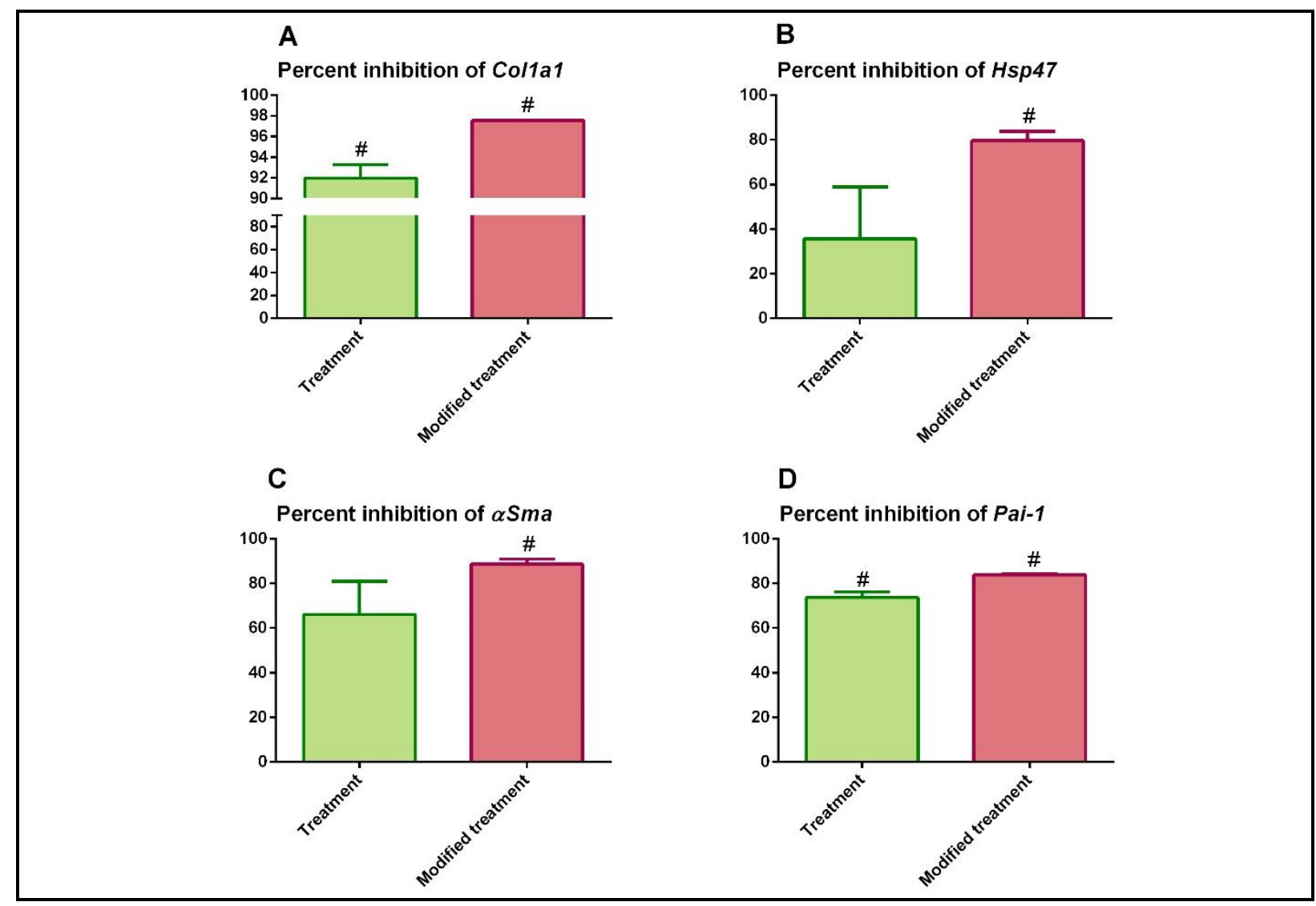

Figure 4. Percent inhibition of relative mRNA expression of fibrosis markers of PCLS in treatment and modified treatment after culture with LY2109761 for 72h. (A) Collagen type I alpha 1 (Collal), (B) heat shock protein 47 (Hsp47), (C) alpha smooth muscle actin $(\alpha \mathrm{Sma})$, and (D) plasminogen-activator inhibitor 1 (Pai-1). Data is expressed as mean + SEM $(\mathrm{n}=3)$. \# $p<0.05$ when compared to corresponding controls.

\subsection{Expression of intracellular signaling protein}

Next, the effect of modified protocol on the expression of intracellular signaling protein of TGF- $\beta$, namely pSmad 2 and tSmad2, was determined. We found that following $72 \mathrm{~h}$ of culture, the level of pSmad2 was 0.7-fold and 0.4fold compared to those before the culture $(0 \mathrm{~h})$ in control and modified control, respectively (Figure 5A, B). In contrast, the level of tSmad2 was 0.7fold and 0.8 -fold compared to $0 \mathrm{~h}$ in control and modified control, respectively (Figure 5A, C). Therefore, it appeared that the modified protocol was not dramatically altered the expression of pSmad2 and tSmad2.

Regarding the effect of anti-fibrotic drug, we found that pSmad2 of treatment and modified treatment were $60 \%$ and $50 \%$ of control and modified control, respectively (Figure 5A, D). In contrast, tSmad2 was only slightly decreased by $31 \%$ in treatment and $23 \%$ in modified treatment when compared to control and modified control, respectively (Figure 5A, E). Thus, we demonstrated that LY2109761 exhibited anti-fibrotic effect in PCLS via the inhibition of Smad2 phosphorylation with less impact on the level of total Smad2 protein expression, irrespective of the culture protocols.

\section{DISCUSSION}

Although PCLS have been routinely used for evaluating anti-fibrotic efficacy of putative compounds, we speculated that characteristics of PCLS-cultured with the conventional protocol may be optimized for testing anti-fibrotic drugs. As expected, this modified culture protocol altered tissue viability and spontaneous fibrogenesis which probably influenced experimental results.

Tissue viability of PCLS-cultured by the modified protocol seemed to be slightly decreased when compared to the conventional protocol for application in drug metabolism and toxicity. Thus, this modified protocol may not be appropriate for a long-term study such as in studying chronic drug metabolism or transport 


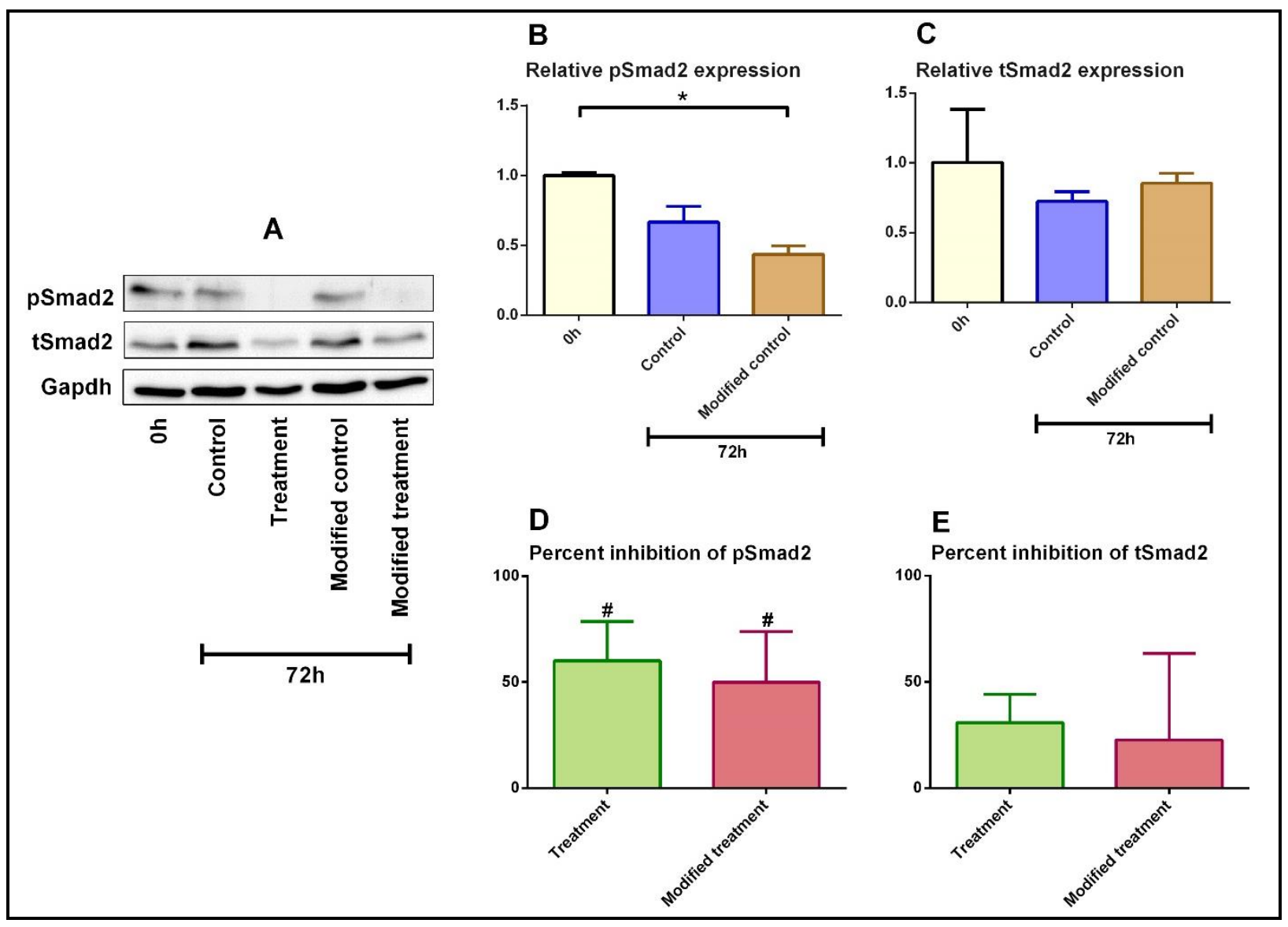

Figure 5. Relative level of phosphorylated Smad2 (pSmad2) and total Smad2 (tSmad2) of PCLS in conventional and modified culture protocol for 72h. (A) Representative set of Western blots, (B) level of pSmad2, (C) level of tSmad2, (D) percent inhibition of pSmad2 after the treatment with LY2109761, and (E) percent inhibition of tSmad2 after the treatment with LY2109761. The levels of pSmad2 were normalized to tSmad2 whereas the levels of tSmad2 were normalized to Gapdh. Data is expressed as mean $+\operatorname{SEM}(\mathrm{n}=3)$. $* p<0.05$ when compared to $0 \mathrm{~h}$ and $\# p<0.05$ when compared to corresponding controls.

functions due to the relatively limited viability of tissue ${ }^{10-12}$. However, ATP/protein of PCLScultured by the modified protocol up to $72 \mathrm{~h}$ was considered viable ${ }^{4,8}$. Furthermore, despite decrease, the amount of isolated RNA from PCLS-cultured by modified protocol was sufficient for downstream processes. Most importantly, the treatment with LY2109761 decreased the viability of PCLS in both conventional and modified protocol almost in the same proportion. Therefore, the assessment of ATP/protein of PCLS-treated with anti-fibrotic drugs to indicate whether the tested candidates elicit toxic effect can be performed by the modified culture protocol.

Spontaneous fibrosis which is a distinctive feature of PCLS was greatly pronounced by the modified culture protocol. Eliminating of the pre-incubation step which can reduce undesirable enzymes and waste products during preparing PCLS could be the major reason why the modified protocol increased the expression of fibrosis markers, and also decreased tissue viability ${ }^{7}$. Spontaneous fibrosis of PCLS is known to be induced by various factors such as intrinsic TGF- $\beta$ activation and oxidative stress from high-glucose and oxygen concentration ${ }^{5-6}$. Interestingly, phosphorylated Smad2 in PCLS-cultured by the modified protocol was slightly lower than that of conventional culture protocol. This finding might indicate that oxidative stress may possible play a major role in spontaneous fibrogenesis beyond intrinsic TGF- $\beta$ activation ${ }^{13,14}$. However, this notion needs to be elucidated further. Marked spontaneous fibrosis in the modified culture protocol can be very useful for evaluating efficacy of anti-fibrotic drugs to mitigate these harmful signaling in fibrogenesis. Nevertheless, in case that exogenous pro-fibrotic substances such as platelet-derived growth factor and lipopolysaccharides will be used to induce pathway-specific fibrogenesis in PCLS, this substantial spontaneous fibrosis may possibly alter the expression profile of fibrosis markers ${ }^{6,15}$. Therefore, the published culture protocol might 
remain a choice for testing the effect of drug against exogenous pro-fibrotic substances.

Noticeably, less deviation among samples by the modified culture protocol of the anti-fibrotic effect of LY2109761 on inhibition of fibrosis gene expression was observed. Definitely, the clear difference in inhibition was mainly due to the increase of spontaneous fibrosis of untreated PCLS. Nevertheless, this finding could largely due to PCLS was exposed to LY2109761 promptly. With published culture protocol, the impact of untreated period on gene expression was the significant up-regulation of HSP47 after culture PCLS prepared form human specimen for only $1 \mathrm{~h}^{5}$. Altogether, these factors would be a reason why the inhibitory effect of LY2109761 on Hsp47 and also $\alpha S m a$ was statistically significance only in the treatment with the modified protocol. Moreover, it should be mentioned that although the sample size of each group in this study is only three $(n=3)$, the statistical testing could still dictate the difference using the modified culture protocol.

Besides differences in tissue viability and spontaneous fibrosis, the modified culture protocol might reduce time and cost of experiment. In the modified culture protocol, plastic plates and culture medium for the preincubation step are not required. Also, once PCLS were treated in the designed condition, the next manipulation with those PCLS is at $24 \mathrm{~h}$, not just $1 \mathrm{~h}$ in the conventional protocol.

Nevertheless, using this modified culture protocol for testing anti-fibrotic drugs in PCLS prepared from human livers is a challenge. Thus far, length to culture human PCLS for testing anti-fibrotic drug with the conventional protocol is limited up to $48 \mathrm{~h}$, the time that spontaneous fibrosis are not dramatically activated ${ }^{4}$. Therefore, prolonged length to culture human PCLS may be the first hurdle to overcome. Lately, human PCLS can be cultured in the conventional protocol up to $120 \mathrm{~h}$ with a change of culture medium ${ }^{16}$; however, using that culture medium for studying anti-fibrotic drug requires further research. Also, the decreased in tissue viability may be expected in human PCLS cultured by the modified protocol.

\section{CONCLUSIONS}

Eliminating pre-incubation step in the culture of PCLS might be a useful alternative method for testing anti-fibrotic drugs. Spontaneous fibrosis which is a distinctive feature of PCLS was greatly pronounced by this modified culture protocol. In addition, eliminating pre-incubation step facilitates immediate exposure of PCLS to anti-fibrotic drug. This novel culture protocol demonstrated the clear inhibitory potency of tested drugs. It should be notified that due to the lack of preincubation step, tissue viability of PCLS appeared to be lower than that of conventional culture protocol. Although, this modified culture protocol remain applicable for assessing toxicity of tested candidates, it might not be appropriate in a long-term study. Besides difference in tissue viability and spontaneous fibrosis, the modified culture protocol might reduce time and cost of experiment.

\section{ACKNOWLEDGEMENTS}

We would like to thank Eric van Haeringen for assisting in the experiments and procedures. We were also supported by Department of Pharmacokinetics, Toxicology and Targeting, University of Groningen, the Netherlands.

\section{Conflict of interest:}

No conflict of interest.

\section{Funding}

ZonMw (grant number 114021010).

\section{Ethical approval}

The study protocol was approved by the Animal Ethical Committee of the University of Groningen.

\section{Article info:}

Received December 2, 2019

Received in revised form February 4, 2020

Accepted February 12, 2020

\section{REFERENCES}

1. Huang X, Cai H, Ammar R, Zhang Y, Wang Y, Ravi K, et al. Molecular characterization of a precision-cut rat liver slice model for the evaluation of antifibrotic compounds. Am J Physiol Gastrointest Liver Physiol. 2019;316(1):G15-24.

2. Olinga P, Schuppan D. Precision-cut liver slices: a tool to model the liver ex vivo. J Hepatol. 2013;58(6):1252-3.

3. Thiele GM, Duryee MJ, Thiele GE, Tuma DJ, Klassen LW. Review: precision cut liver slices for the evaluation of fatty liver and fibrosis. Curr Mol Pharmacol. 2017;10(3):249-54.

4. Luangmonkong T, Suriguga S, Bigaeva E, Boersema M, Oosterhuis D, de Jong KP, et al. Evaluating the antifibrotic potency of galunisertib in a human ex vivo model of liver fibrosis. $\mathrm{Br} \mathrm{J}$ Pharmacol. 2017;174(18):3107-17. 
5. Westra IM, Mutsaers HAM, Luangmonkong T, Hadi M, Oosterhuis D, de Jong KP, et al. Human precision-cut liver slices as a model to test antifibrotic drugs in the early onset of liver fibrosis. Toxicol In Vitro. 2016;35:77-85.

6. Westra IM, Oosterhuis D, Groothuis GM, Olinga P. The effect of antifibrotic drugs in rat precision-cut fibrotic liver slices. PLoS One. 2014;9(4):e95462.

7. de Graaf IA, Olinga P, de Jager MH, Merema MT, de Kanter R, van de Kerkhof EG, et al. Preparation and incubation of precision-cut liver and intestinal slices for application in drug metabolism and toxicity studies. Nat Protoc. 2010;5(9):1540-51.

8. Luangmonkong T, Suriguga S, Adhyatmika A, Adlia A, Oosterhuis D, Suthisisang $C$, et al. In vitro and ex vivo anti-fibrotic effects of LY2109761, a small molecule inhibitor against TGF-beta. Toxicol Appl Pharmacol. 2018;355:127-37.

9. Kilkenny C, Browne WJ, Cuthill IC, Emerson M, Altman DG. Improving bioscience research reporting: the ARRIVE guidelines for reporting animal research. PLoS Biol. 2010;8(6):e1000412.

10. Ferrero JL, Brendel K. Liver slices as a model in drug metabolism. Adv Pharmacol. 1997; 43:131-69.

11. Gandolfi AJ, Wijeweera J, Brendel K. Use of precision- cut liver slices as an in vitro tool for evaluating liver function. Toxicol Pathol. 1996; 24(1):58-61.

12. Glockner R, Steinmetzer P, Drobner C, Muller D. Application of cryopreserved precision-cut liver slices in pharmacotoxicology - principles, literature data and own investigations with special reference to CYP1A1mRNA induction. Exp Toxicol Pathol. 1998;50(4-6): 440-9.

13. Luangmonkong T, Suriguga S, Mutsaers HAM, Groothuis GMM, Olinga P, Boersema M. Targeting oxidative stress for the treatment of liver fibrosis. Rev Physiol Biochem Pharmacol. 2018;175:71-102.

14. Sanchez-Valle V, Chavez-Tapia NC, Uribe M, MendezSanchez N. Role of oxidative stress and molecular changes in liver fibrosis: a review. Curr Med Chem. 2012;19(28):4850-60.

15. Olinga P, Merema MT, de Jager MH, Derks F, Melgert $\mathrm{BN}$, Moshage $\mathrm{H}$, et al. Rat liver slices as a tool to study LPS-induced inflammatory response in the liver. J Hepatol. 2001;35(2):187-94.

16. Starokozhko V, Vatakuti S, Schievink B, Merema MT, Asplund A, Synnergren J, et al. Maintenance of drug metabolism and transport functions in human precisioncut liver slices during prolonged incubation for 5 days. Arch Toxicol. 2017;91(5):2079-92. 